\title{
ARQUITECTURA FOLIAR Y ANATOMÍA DE LA CORTEZA Y LA MADERA DE QUERCUS SARTORII Y Q. XALAPENSIS (FAGACEAE)
}

\author{
Dorismildda Martínez-Cabrera ${ }^{1}$, Teresa Terrazas ${ }^{1}$ y Fernando Zavala-Chávez ${ }^{2}$ \\ 1Programa en Botánica, Colegio de Postgraduados, Montecillo, Estado de México, 56230, México. \\ Correo-e: winchi@colpos.mx \\ ${ }^{2}$ División de Ciencias Forestales, Universidad Autónoma Chapingo, Chapingo, Estado de México \\ 56230, México.
}

\begin{abstract}
Resumen: En este trabajo se describe y compara la arquitectura foliar y la anatomía de la corteza y la madera de Quercus sartorii y Q. xalapensis, especies morfológicamente similares, en la búsqueda de caracteres que contribuyan a su delimitación. Los resultados mostraron que los caracteres del patrón de venación: número y forma de aréolas, ramificación de las vénulas y recorrido de la vena secundaria dentro del diente son caracteres que permiten distinguir a las especies, no así el tamaño de la hoja y el número de los estomas. Las especies difieren en la corteza externa y la felodermis. La madera de ambas especies es similar a lo reportado para el género, pero se observó una distribución diferencial de los vasos, menos abundantes y solitarios, en hileras radiales en $Q$. sartorii. Los atributos mencionados deberán emplearse en conjunto con caracteres de la flor, el fruto y la semilla para apoyar el reconocimiento de ambas especies.
\end{abstract}

Palabras clave: felodermis, floema, patrón de venación, peridermis, xilema.

\begin{abstract}
Leaf architecture and wood and bark anatomy of Quercus sartorii and Q. xalapensis, species with similar morphology, are described and compared, with the main purpose of recognizing features that may contribute to distinguish them. The results showed that venation pattern features such as areole number and shape, veintlets branching, and secondary vein arrangement inside the tooth are characters that help distinguish both species, but not leaf size and stomata number. Both species differ in their external bark appearance and phelloderm. Wood of both species is similar to that reported for the genus, but there is a tendency to having fewer vessels and solitary in radial rows in $Q$. sartorii. These traits should be used together with floral, fruit and seed features to support the recognition of both species.
\end{abstract}

Key words: periderm, phelloderm, phloem, venation pattern, xylem.

$\mathbf{E}$ género Quercus incluye árboles y arbustos con alrededor de 500 especies (Nixon, 1989), dominantes en una amplia diversidad de hábitats en el Hemisferio Norte. En México este género presenta alrededor de 125-150 especies (ZavalaChávez, 1995) comprendidas en las secciones Lobatae (encinos rojos), Quercus (encinos blancos) y Protobalanus (encinos intermedios), de las cuales 86 son endémicas de México (Nixon, 1993). Las especies mexicanas del género Quercus se encuentran distribuidas desde climas cálidos hasta templados donde son codominantes junto con los pinos, constituyendo la mayor parte de la cubierta vegetal de estos lugares (Rzedowski, 1978). Existen varios pares de especies de Quercus en México que debido a su variación morfológica tienen problemas de delimitación, por ejemplo $Q$. acutifolia Neé y $Q$. conspersa Benth., $Q$. affinis Scheidw. y $Q$. laurina Humb. et Bonpl., $Q$. canbyi Trel. y $Q$. albocinata Trel., $Q$. laeta Liebm. y $Q$. obtusata Humb. et Bonpl., Q. mexicana Humb. et Bonpl. y Q. crassipes Humb. et Bonpl., Q. sartorii Liebm. y $Q$. xalapensis Humb. et Bonpl., y $Q$. uxoris
McVaugh y $Q$. skinneri Benth. (Valencia y Delgado 1995; Zavala-Chávez, 1995; Romero et al., 2000; Martínez-Cabrera et al., en prensa).

Los estudios de plantas actuales y fósiles indican que la arquitectura foliar, en especial los patrones de venación, es valiosa en la determinación de las relaciones taxonómicas y filogenéticas entre las angiospermas (Hickey y Wolfe, 1975). Los trabajos de arquitectura foliar en el género Quercus son escasos, pero han mostrado que existe variación discreta útil para la distinción de algunas especies (Valencia y Delgado, 1995; Romero, 2000), en especial el indumento y sus varios tipos (Hardin, 1979; Manos, 1993; Llamas et al., 1995). Las investigaciones realizadas hasta la fecha con anatomía de la corteza han probado que ésta puede ser una herramienta valiosa en los estudios sistemáticos (Bamber, 1962; Whitmore, 1962, 1963; Roth, 1981; Van Wyk, 1985; Trockenbrodt y Parameswaran, 1986; Ritcher y Van Wyk, 1990; Terrazas, 1995; Orduño-Cruz y Terrazas, 1998), pero son escasos los trabajos comparativos para este tejido en el 
género Quercus (Whitmore, 1963; Howard, 1977). Se ha documentado que los caracteres anatómicos de la madera contribuyen a la ubicación de los taxa en diferentes categorías taxonómicas (Dickison, 1975; Stuessy, 1990). La anatomía sistemática proporciona evidencias para apoyar o refutar la ubicación de individuos en su respectiva especie (Baas, 1982; Chalk, 1983; Stuessy 1990; Terrazas y Wendt, 1995). En el género Quercus se han realizado trabajos que describen los caracteres anatómicos de la madera y su relación con las propiedades físico-mecánicas (Corral, 1981; de la Paz, 1974, 1982, 1985; de la Paz et al., 1998). No obstante, son pocas las investigaciones que utilizan los caracteres anatómicos para separar especies o grupos de especies en el género Quercus (de la Paz y Aguilar, 1978; Valencia y Barajas-Morales, 1995). Valencia y Barajas-Morales distinguen a $Q$. affinis de $Q$. laurina por medio de las diferencias anatómicas de su madera.

Con base en lo anterior se describe y se compara la arquitectura foliar y la anatomía de la corteza y de la madera de Quercus sartorii y $Q$. xalapensis, par de especies cuya variación morfológica dificulta su delimitación, con la finalidad de encontrar atributos anatómicos que apoyen o refuten su distinción como especies diferentes.

\section{Materiales y métodos}

Se recolectaron 47 individuos provenientes de 11 poblaciones de Quercus sartorii y Q. xalapensis de los estados de Hidalgo, San Luis Potosí, Tamaulipas y Veracruz (cuadro 1). En dos localidades del estado de Tamaulipas, ambas especies se encontraron en simpatría (cuadro 1). Las recolectas incluyen el municipio de Xalapa, Veracruz, señalado como la localidad tipo de $Q$.xalapensis y de $Q$. xalapensis $\mathrm{f}$. jalapae (Trelease, 1924). Para el estudio de la anatomía de la corteza y la madera se cortaron ramas de $2 \mathrm{~cm}$ de diámetro de dos individuos por población. También se removieron hojas completas y maduras de 45 ejemplares de los herbarios CHAPA, ENCB, MEXU y XAL (apéndice 1) para ampliar la muestra y describir la arquitectura foliar.

Las hojas se diafanizaron con $\mathrm{NaOH}$ al $5 \%$ por $48 \mathrm{~h}$, se lavaron y se agregó hipoclorito comercial al 50\% por $6 \mathrm{~h}$. Enseguida, fueron deshidratadas con una serie de alcoholes (50, 70 y $96 \%)$, se tiñeron con safranina en alcohol al $96 \%$, se terminaron de deshidratar en dos cambios de alcohol $(100 \%)$ y tres de xilol y se montaron con resina sintética (Dilcher, 1974). La descripción de la arquitectura foliar se realizó con base en la terminología propuesta por Hickey (1973, 1974). Para cinco caracteres (largo y ancho de la hoja, número de estomas $/ \mathrm{cm}^{2}$, longitud de las células oclusivas y número de aréolas $/ \mathrm{cm}^{2}$ ) se evaluó la existencia de diferencias significativas entre especies por medio de un análisis de varianza (SAS Institute, 1989).

Las muestras recolectadas para el estudio de la corteza y la madera se fijaron en glicerina-alcohol-agua (1:1:1) en el campo. Se hicieron cortes transversales de $25-30 \mu \mathrm{m}$ de espesor con un micrótomo de deslizamiento. Los cortes se tiñeron con safranina-verde rápido y se montaron con resina sintética (Johansen, 1940). La descripción macroscópica de la corteza se realizó utilizando la terminología de Junikka (1994) y la descripción microscópica con la terminología propuesta por Trockenbrodt (1990). La madera se describió siguiendo las recomendaciones de la Asociación Internacional de Anatomistas de la Madera (IAWA Committee, 1989).

\section{Resultados}

Arquitectura foliar. Quercus sartorii y $Q$. xalapensis tienen hojas lanceoladas, elípticas u ovadas con margen dentado, dientes aristados, base aguda, obtusa o cordada y ápice agudo o acuminado (figuras 1 y 2). El tamaño de las hojas es de $13.7 \pm 1.3 \mathrm{~cm}$ de largo y $4.6 \pm 0.5 \mathrm{de}$ ancho en $Q$. sartorii y

Cuadro 1. Localización geográfica de las poblaciones recolectadas de Quercus xalapensis y Q. sartorii en la República Mexicana, nombre del colector y número de colecta de los ejemplares depositados en CHAPA. *Localidad tipo para Q. xalapensis y Q. xalapensis f. jalapae.

\begin{tabular}{lll}
\hline Estado & Localidad & Especie \\
\hline Hidalgo & Lontla, Mpio. Tlanchinol & Q. xalapensis Martínez 153-157 \\
& Tlahuelompa, Mpio. Zacualtipán & Q. sartorii Martínez 158-162 \\
San Luis Potosí & San Antonio, Mpio. Xilitla & Q. xalapensis Martínez y Zavala 150-152 \\
Tamaulipas & Q. xalapensis Martínez y Zavala 134-137; \\
& San José, Mpio. Gómez Farías & Q. sartorii Martínez y Zavala 138-141 \\
& & Q. xalapensis Martínez y Zavala 142-145; \\
Peracruz & Parque F. J. Clavijero, Mpio. Xalapa* & Q. sartorii Martínez y Zavala 146-149 \\
& Rarranca Grande, Mpio. Xico & Q. xalapensis Martínez 119-123 \\
& Rancho La Mesánez y Zavala 116-118 & Q. xalapensis Martínez 129-133 \\
& Rancho La Hierbabuena, Mpio. San Andrés & Q. xalapensis Martínez 124-128 \\
& Tlalnehuayocan & \\
\hline
\end{tabular}




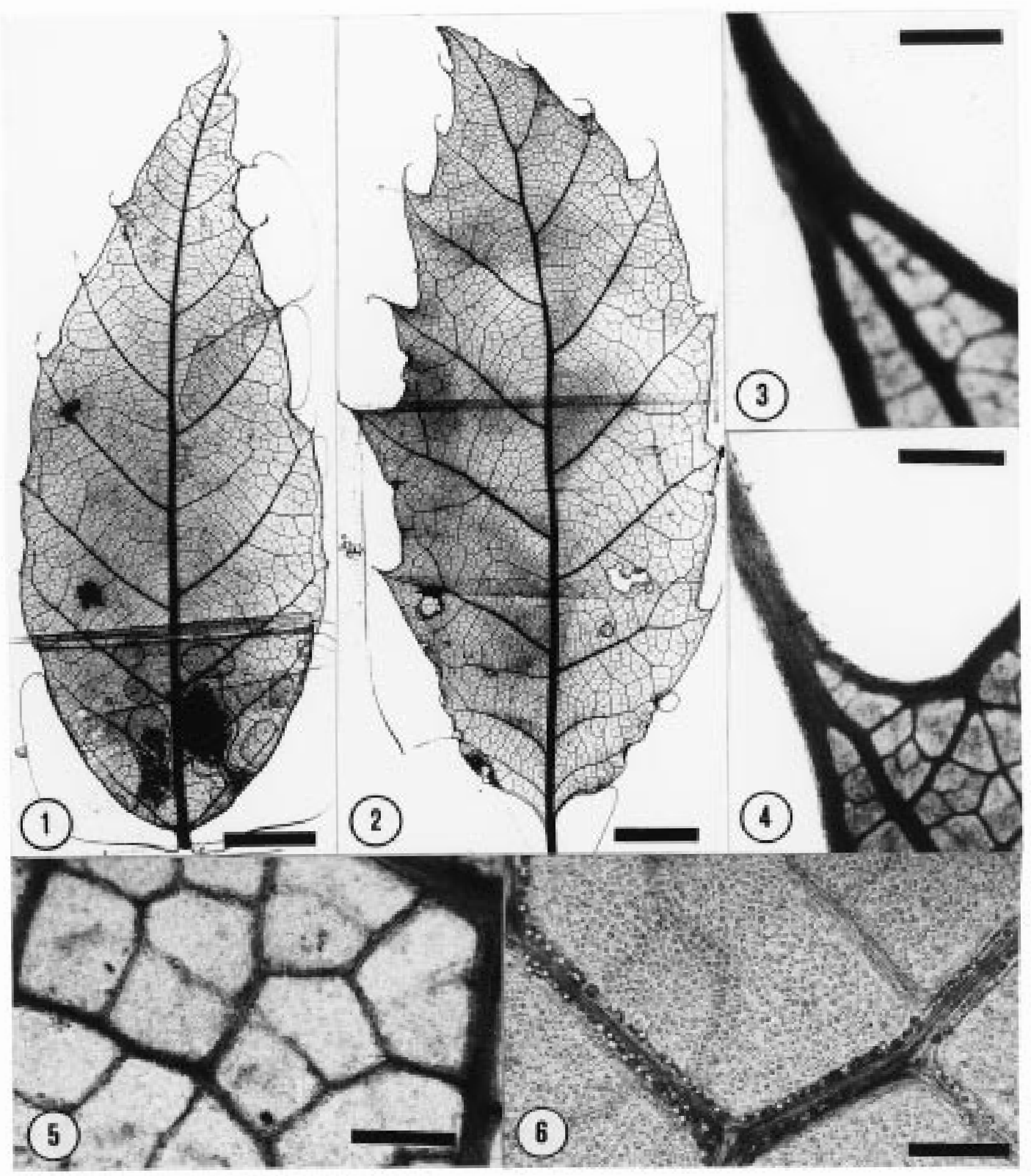

Figuras 1-6. Arquitectura foliar. 1. Hoja de Q. sartorii, Martínez 158 (CHAPA). 2. Hoja de Q. xalapensis, Martínez 120 (CHAPA). 3. Vena secundaria con curso central Q. xalapensis, J. Rzedowski 18931 (MEXU). 4. Vena secundaria con curso excéntrico Q. sartorii, Durán 1082 (MEXU). 5. Patrón ortogonal Q. sartorii, Martínez 13 (CHAPA). 6. Cristales de oxalato de calcio en venas de 3er y 4o orden. Q. xalapensis, Martínez 159 (CHAPA). Escala: 1-2 = $1 \mathrm{~cm}, \mathbf{3 - 4}=500 \mu \mathrm{m}, \mathbf{5}=200 \mu \mathrm{m}, \mathbf{6}=100 \mu \mathrm{m}$. 
Cuadro 2. Síntesis del análisis de varianza para cinco caracteres de la arquitectura foliar y comparación de medias entre las especies estudiadas. Letras iguales indican que no existen diferencias estadísticamente significativas $(P<0.05)$.

\begin{tabular}{lccc}
\hline \multicolumn{1}{c}{ Carácter } & Valores de $\mathrm{F}$ y probabilidad & Q. sartorii & Q. xalapensis \\
\hline Largo de la hoja $(\mathrm{cm})$ & $F=2.16$, g.I. $=1, P>0.14, \mathrm{~N}=92$ & $13.7 \pm 1.3^{\mathrm{a}}$ & $14.2 \pm 1.8^{\mathrm{a}}$ \\
Ancho de la hoja $(\mathrm{cm})$ & $F=0.51$, g.I. $=1, P>0.47, \mathrm{~N}=92$ & $4.6 \pm 0.5^{\mathrm{a}}$ & $4.7 \pm 0.7^{\mathrm{a}}$ \\
Longitud del estoma $(\mu \mathrm{m})$ & $F=0.34$, g.I $=1, P>0.56, \mathrm{~N}=92$ & $23 \pm 4.1^{\mathrm{a}}$ & $23 \pm 3.3^{\mathrm{a}}$ \\
Estomas $/ \mathrm{cm}^{2}$ & $F=2.31$, g.I. $=1, P>0.13 \mathrm{~N}=92$ & $397 \pm 70.8^{\mathrm{a}}$ & $377 \pm 52.7^{\mathrm{a}}$ \\
Areolas $/ \mathrm{cm}^{2}$ & $F=172.9$, g.I. $=1, P<0.0001, \mathrm{~N}=92$ & $22 \pm 4.8^{\mathrm{a}}$ & $12 \pm 2.3^{\mathrm{b}}$ \\
\hline
\end{tabular}

de $14.2 \pm 1.8 \mathrm{~cm}$ de largo y $4-7 \pm 0.7 \mathrm{~cm}$ de ancho en $Q$. xalapensis; no se detectaron diferencias estadísticamente significativas para estos atributos (cuadro 2).

Las hojas tienen una nervadura semicraspedódroma; la vena primaria no presenta ramificaciones, sino que sigue un recorrido recto y muestra un tamaño moderadamente grueso (figuras 1 y 2). Las venas secundarias también son moderadamente gruesas y surgen de la vena primaria, formando ángulos de divergencia agudos con un intervalo de $45^{\circ}$ a $80^{\circ}$; el ángulo de divergencia disminuye hacia el ápice de la hoja. El recorrido de estas venas es de recto a ligeramente curvo. Cada vena secundaria se ramifica en tres, a diferentes distancias del margen, formando ángulos casi rectos. La parte central entra al diente por la parte media y presenta un recorrido central en $Q$. xalapensis (figura 3), mientras que en $Q$. sartorii el recorrido es excéntrico debido a que se une al margen de la lámina foliar (figura 4). En ambas especies, esta parte de la vena secundaria termina en un diente de tipo aristado; las otras dos ramas de la vena se unen a otras venas secundarias formando ángulos obtusos. Las venas de tercer orden se originan en ángulo recto con respecto a las secundarias; su ramificación es transversal, con un patrón reticulado-ortogonal (figura 5) y el recorrido es, en ocasiones, moderadamente sinuoso. Las venas de cuarto y quinto orden forman aréolas completas de tipo ortogonal; el número de aréolas en Q.xalapensis es de 10 a $14 / \mathrm{cm}^{2}$ y en Q. sartorii de 17 a $27 / \mathrm{cm}^{2}$, encontrándose diferencias estadísticamente significativas entre especies para este carácter (cuadro 2). En Q. xalapensis las vénulas son predominantemente ramificadas, una o dos veces, y muy raramente se detectan simples, mientras que en $Q$. sartorii predominan las vénulas simples, lineares o curvas y casi nunca se observan una vez ramificadas. La vena última marginal es incompleta o forma arcos. La vena principal presenta cristales romboédricos en toda la superficie y las venas de 2o, 3er y 40 orden presentan los cristales únicamente a los lados de éstas (figura 6).

Superficie foliar. En la superficie se observan tricomas estrellados y simples. Los estomas anomocíticos tienen una longitud de $23 \mu \mathrm{m}$ en ambas especies y se distribuyen al azar en la superficie abaxial, siendo de 326 a $468 / \mathrm{cm}^{2}$ en $Q$. sartorii y de 324 a $430 / \mathrm{cm}^{2}$ en $Q$. xalapensis. Aunque hay una tendencia en $Q$. sartorii a presentar una mayor número de estomas, no se detectaron diferencias significativas entre especies (cuadro 2).

Descripción macroscópica de la corteza. En Quercus xalapensis la corteza es fisurada a ligeramente escamosa, de color café oscuro, tamaño regular y forma rectangular, en líneas ligeramente diagonales; la corteza interna es de color rojizo. En $Q$. sartorii la corteza es escamosa, de color gris o algunas veces café oscuro, con escamas gruesas, generalmente de tamaño y forma irregular; la corteza interna es de color rosa pálido.

\section{Descripción microscópica del floema secundario.}

- Elementos de tubo criboso (ETC) y células acompañantes (CA). Los ETC en el floema no colapsado se encuentran en grupos de 4 a 6 células en Quercus sartorii, mientras que en $Q$. xalapensis son de 4 a 8 células, difusos entre células del parénquima axial; su pared es delgada y tienen forma redonda o angulosa. En ambas especies, se encuentran una o dos CA por ETC, situadas en las esquinas o a los lados de los ETC y son de pared delgada y de forma triangular o redonda (figura 7). En el floema colapsado, los ETC y CA se obliteran irregularmente.

- Parénquima axial. En ambas especies se encuentra difuso entre los ETC y CA; comúnmente se interrumpe por grupos de fibras y radios floemáticos; son células de pared delgada, de forma angular o redonda y generalmente contienen cristales prismáticos, especialmente en los márgenes de las bandas de fibras y cristales tipo drusa de diferentes tamaños (figura 7). Algunas de estas células con cristales se encuentran cerca del cámbium vascular y otras, sin contenidos, se colapsan al alejarse del cámbium vascular.

- Parénquima radial. El curso de los radios es recto, rara vez se colapsan al alejarse del cámbium vascular. Las células de los radios floemáticos presentan cristales romboédricos (figura 8).

- Esclerénquima. En ambas especies las fibras se encuentran 


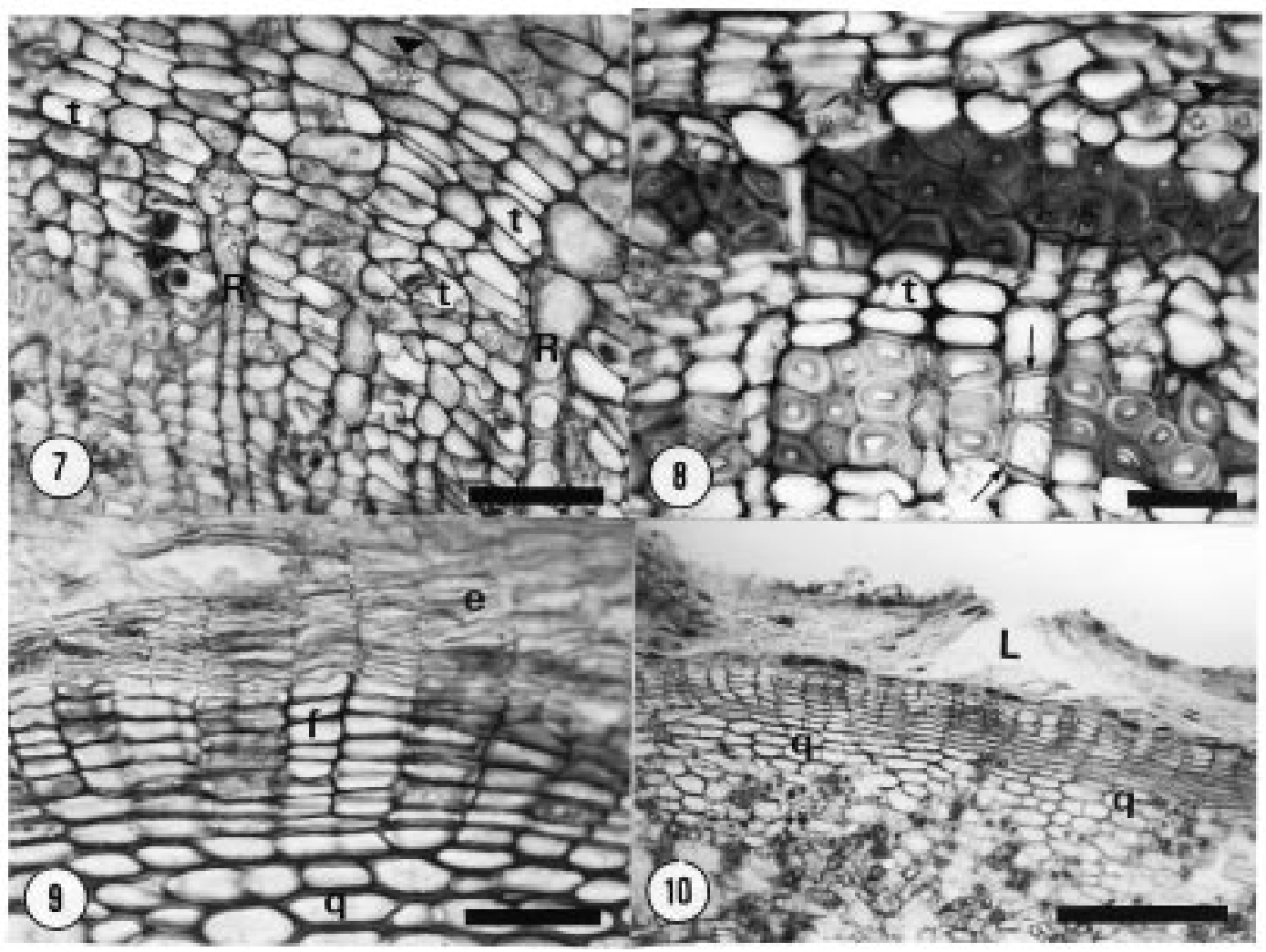

Figuras 7-10. Corteza cortes transversales. 7. Floema no colapsado con tubos cribosos y células acompañantes, drusas en células de parénquima (flechas) Q. xalapensis, Martínez 119 (CHAPA). 8. Bandas de esclerénquima con fibras gelatinosas y cristales prismáticos en células de radio floemático Q. sartorii, Martínez 146 (CHAPA). 9. Felodermis $Q$. xalapensis, Martínez 126 (CHAPA). 10. Lenticela. $Q$. xalapensis, Martínez 129 (CHAPA). Escala: 7, 9-10 =50 $\mu \mathrm{m}, 8=30 \mu \mathrm{m}$; e = felema, $\mathrm{f}=$ felodermis, $\mathrm{L}=$ lenticela, $\mathrm{q}=$ colénquima, $\mathrm{R}=$ radio floemático, $\mathrm{t}=$ tubo criboso.

en grupos, formando junto con las esclereidas una banda tangencial. La pared de las fibras es gruesa (figura 8) y a veces del tipo gelatinoso. Las esclereidas tienen forma isodiamétrica y pared gruesa con punteaduras simples. Se encuentran junto con las fibras formando una banda continua, que generalmente está formada por 2 a 4 células de espesor.

- Peridermis. La peridermis está constituida por felema, felógeno y felodermis. El felógeno tiene de 1 a 2 estratos de células de pared delgada y forma rectangular, y se origina de las células subepidérmicas. En Quercus sartorii la felodermis está comúnmente ausente, pero cuando se presenta tiene de 1 a 3 estratos de células de forma rectangular o cuadrada y de paredes delgadas, que presentan en su lumen celular granos de almidón y taninos. En Q. xalapensis la felodermis se compone por lo general de 4 a 10 estratos de células (figura 9); las células de los estratos más cercanos al felógeno tienen forma rectangular y contienen en su lumen celular taninos; los estratos más internos adquieren forma irregular, perdiendo su disposición, y en su lumen contienen drusas. El felema se compone de células rectangulares, de paredes delgadas, tamaño variable y los taninos ocluyen su lumen; únicamente se interrumpen en las lenticelas, donde se observa tejido de relleno con células de paredes más delgadas (figura 10). El ritidoma no se presenta en las ramas estudiadas.

- Córtex. Debido a que los cortes se realizaron en ramas de $2 \mathrm{~cm}$ de diámetro, también se observa la presencia de córtex. En ésta se presenta una región de colénquima formada por 3 a 6 estratos de células; estas células son de forma alargada cuando están cerca de la peridermis (figuras 9 y 10) y redonda cuando se alejan de ésta. Bajo esta región de colénquima se encuentra una región formada por células de parénquima con abundantes drusas (figura 10) y en Quercus xalapensis se observan esclereidas aisladas o formando grupos.

Madera. La madera de ambas especies tiene porosidad semianular. En Quercus sartorii los vasos son exclusivamente 


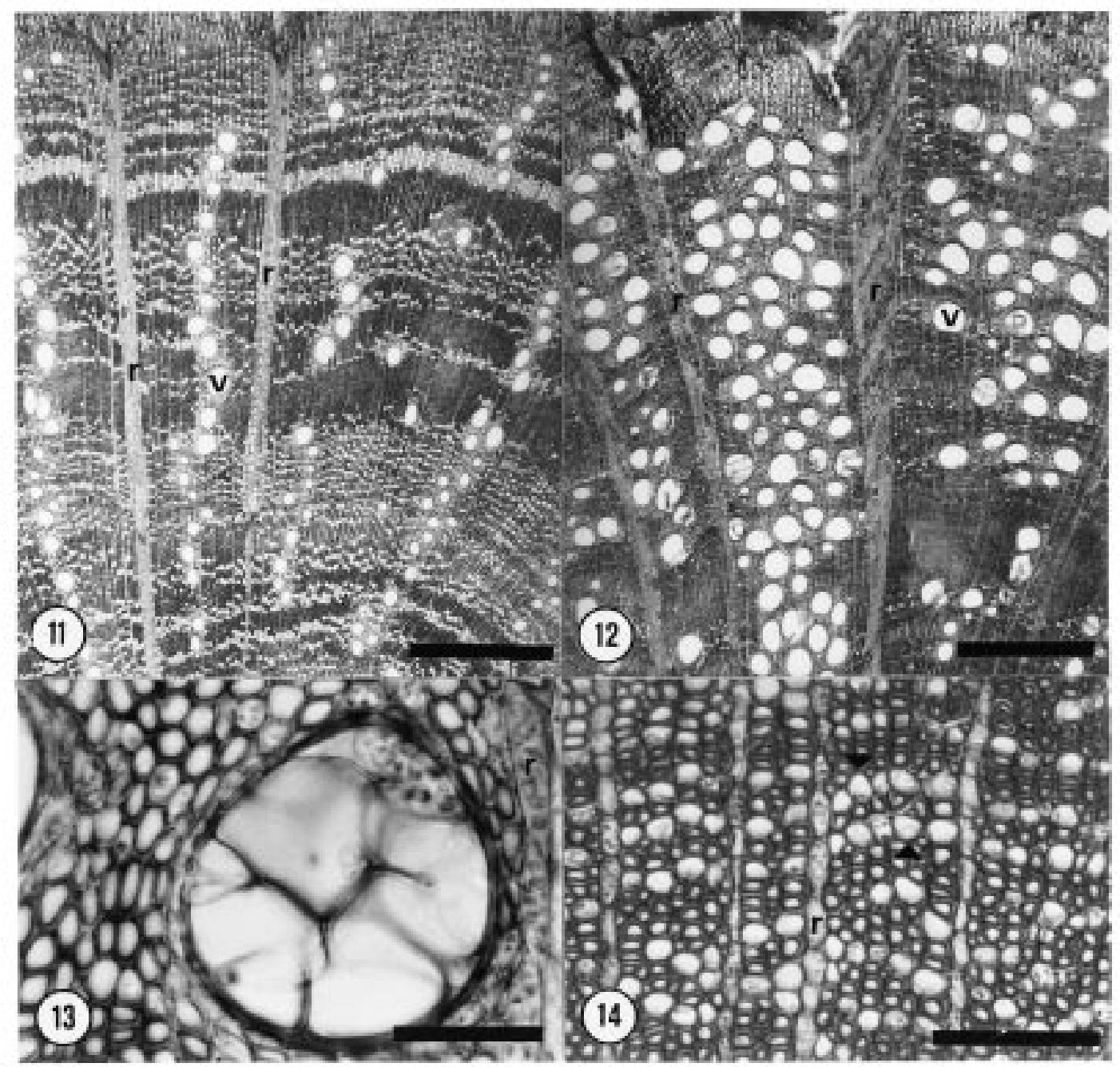

Figuras 11-14. Madera cortes transversales . 11. Vasos solitarios, escasos y en hileras radiales uniseriadas, $Q$. sartorii, Martínez 138 (CHAPA). 12. Vasos solitarios, abundantes y sin arreglo aparente, Q. xalapensis, Martínez 142 (CHAPA). 13. Tílides en vaso, Q. xalapensis, Martínez 170 (CHAPA). 14. Parénquima apotraqueal (flechas), Q. xalapensis, Martínez 119 (CHAPA). Escala: 11-12 = 500 $\mu \mathrm{m}, 13-14$ = $100 \mu \mathrm{m} ; \mathrm{r}=$ radio, $\mathrm{v}=$ vaso.

solitarios y de forma circular, arreglados en hileras radiales uniseriadas y oblicuas (figura 11); en $Q$. xalapensis también predominan los vasos solitarios pero son más abundantes, sin un arreglo definido y en ocasiones aparentan formar grupos (figura 12). Ambas especies presentan tílides en algunos vasos de mayor diámetro (figura 13) y están ausentes en los vasos de menor diámetro.

- Parénquima axial. Las dos especies tienen parénquima apotraqueal difuso y en bandas (figura 14). Estas bandas de parénquima en Quercus sartorii son de 1 a 4 estratos de células (figura 11) y en $Q$. xalapensis se presentan comúnmente de un estrato (figura 14).

- Radios. Se presentan dos tipos, los uniseriados que son los más comunes, y los multiseriados que en Quercus sar- torii tienen una anchura de 2 a 6 células, en corte transversal (figura 11) y en Q. xalapensis son de 3 a 10 células de anchura y más abundantes (figura 12). Los radios de ambas especies contienen cristales de forma romboidal y algunas drusas.

- Fibras. En ambas especies, las fibras tienen pared gruesa y lumen reducido y también se presentan abundantes fibras gelatinosas.

\section{Discusión}

La arquitectura foliar de Quercus sartorii y $Q$. xalapensis es similar, ya que comparten caracteres de la forma, el tamaño y el patrón de venación. Trelease (1924) utiliza la forma y el 
tamaño de las hojas junto con el tamaño de los frutos para hacer la distinción entre estas dos especies. Sin embargo, el tamaño y forma de la hoja no permitieron separar a $Q$. sartorii de $Q$. xalapensis; por ello es difícil reconocerlas en ejemplares de herbario estériles. Martínez et al. (en prensa) confirman que el tamaño del fruto y el tiempo de maduración de éstos separan ambas especies. Los tricomas observados fueron estrellados y simples, en ambas especies, y a diferencia de otros pares de especies como $Q$. acutifolia - $Q$. conspersa, $Q$. crassipes - Q. mexicana, Q. repanda - Q. microphylla y Q. skinerii - Q. uxoris (Zavala-Chávez, 1995; Romero, 2000), no permiten separarlas. El número de estomas tampoco contribuyó a separar las especies estudiadas. No obstante, los atributos del patrón de venación como número y forma de las aréolas, ramificación de las vénulas y recorrido de la vena secundaria dentro del diente separaron a los individuos de $Q$. sartorii de los de Q. xalapensis. Quercus sartorii presentó un mayor número de aréolas por $\mathrm{cm}^{2}$ y vénulas simples, ya sea rectas o curvas, en comparación con $Q$. xalapensis con un menor número de áreolas $/ \mathrm{cm}^{2}$ y vénulas que se ramifican una o dos veces. El tipo de diente que presentan ambas especies fue aristado y las venas de tercer orden marcadamente transversales. La descripción morfológica de este tipo de diente y las venas de tercer orden coinciden con la descripción de Hickey y Wolfe (1975) para el orden Fagales. Sin embargo, el recorrido que presenta la vena secundaria dentro del diente fue diferente; en $Q$. sartorii es excéntrico y en $Q$. xalapensis el recorrido es central. Este carácter es considerado de valor diagnóstico (Hickey y Wolfe, 1975). Hickey y Wolfe señalan que, en general, la morfología del diente posee valor taxonómico a nivel específico.

Los resultados de este trabajo confirmaron la contribución de los caracteres del patrón de venación en la distinción de especies de encinos como habían señalado Valencia y Delgado (1995). Romero (2000) resalta la importancia de la arquitectura foliar en encinos y señala que son escasos los trabajos con este enfoque en el género Quercus, por lo que sugiere que el patrón de venación podría contribuir parcialmente para resolver problemas taxonómicos a nivel específico, aseveración que apoyamos con este trabajo. Para el par de especies estudiado ni la variación del tamaño de la hoja, ni el tipo de tricomas o el número de estomas permitió separarlas.

Los individuos de Quercus sartorii y Q.xalapensis difieren en los caracteres macroscópicos de la corteza, principalmente en el tamaño y color de las escamas. Sin embargo, comparten caracteres anatómicos de la corteza. En el floema se observó una banda tangencial de esclereidas y fibras. Esta descripción coincide con lo señalado para $Q$. robur L. (Trockenbrodt, 1995b) y 11 especies de Quercus del sureste de los Estados Unidos (Howard, 1977). Otros estudios también mencionan que esta banda de esclerénquima forma parte de la corteza de varias especies de la familia Fagaceae, como Fagus sylvatica L. y Castanea sativa Mill. (Whitmore, 1963), así como de algunas especies de la familia Dipterocarpaceae, de la cual difieren únicamente en la cantidad de fibras (Whitmore, 1962). Quercus sartorii y Q. xalapensis presentaron cristales romboédricos en las células de parénquima, en los márgenes de las bandas de esclerénquima y drusas en las células del parénquima floemático y del córtex. Varios autores han mencionado que ambos tipos de cristales se presentan en otras especies del género Quercus (Howard, 1977; Cuttler et al., 1987; Trockenbrodt, 1995a).

La peridermis en ambas especies tuvo su origen bajo la epidermis, de la primera hilera de células del córtex. El origen subepidérmico del felógeno ha sido descrito para numerosas especies (Roth, 1981) y en particular para varias especies de encinos rojos y blancos (Howard, 1977; Trockendrodt, 1995b). En la peridermis se encontró un carácter contrastante entre las especies estudiadas. La felodermis en Quercus sartorii está comúnmente ausente, pero cuando se presenta se forman de 1 a 3 estratos de células, siendo más frecuente la presencia de dos estratos. En contraste, Q. xalapensis presenta una felodermis bien desarrollada con 4 a 10 estratos de células. Se sugiere estudiar un mayor número de especies con objeto de confirmar la homogeneidad en el floema secundario del género señalada por Howard (1977), así como para corroborar la diferencia en el número de estratos de la felodermis entre las dos especies estudiadas al compararla con otras especies de Quercus.

La madera de los individuos de Quercus sartorii y $Q$. xalapensis presentaron elementos de vaso solitarios, pero el arreglo de éstos fue diferente. En $Q$. sartorii los vasos se arreglaron en hileras radiales uniseriadas, largas y oblicuas, pero en $Q$. xalapensis no presentaron ningún arreglo o formaron grupos aparentes. Es interesante mencionar que la madera descrita para $Q$. sartorii (Corral, 1981) y Q. xalapensis (de la Paz et al., 1998) de individuos adultos, también presenta la distribución diferencial de los vasos aquí señalada para las ramas de $2 \mathrm{~cm}$ de diámetro, lo que permite sugerir que la madera de estas especies se distingue por este atributo. La presencia de escasas tílides en $Q$. sartorii y $Q$. xalapensis coincide con lo descrito para el subgénero Lobatae al que pertenecen (de la Paz, 1976, 1982; Panshin y de Zeeuw, 1980; Corral, 1981; de la Paz et al., 1998). La presencia de tílides escasas ha sido señalada por de la Paz (1982) como un atributo contrastante entre los subgéneros Lobatae y Quercus, porque las tílides son abundantes en la madera de los encinos blancos (Quercus). Las dos especies presentaron parénquima apotraqueal de distribución difusa y en bandas con algunos cristales romboidales o drusas. Este arreglo del parénquima es considerado como un carácter común en los encinos rojos (Panshin y de Zeeuw, 1980), que varios autores denominan apotraqueal en agregados o reticulado con base en su abundancia (Corral, 1981; de la Paz et al., 1998). Dos Santos y Miller (1998) mostraron que existen atributos anatómicos en la madera que no se ven afectados por el desarrollo del 
cámbium vascular, como los resultados sugieren para la distribución diferencial de los vasos entre las especies de Quercus estudiadas.

Los caracteres de la arquitectura foliar como el número y la forma de aréolas, la ramificación de las vénulas y el recorrido del nervio secundario dentro del diente, contribuyen a la separación de las especies estudiadas. Estos caracteres son contrastantes aun en los individuos provenientes de las poblaciones simpátricas. La apariencia externa de la corteza y los caracteres anatómicos en la peridermis (número de células de la felodermis) y en la madera (distribución de los vasos) también permitieron separar a los individuos de Quercus sartorii y $Q$. xalapensis estudiados. Los caracteres aquí encontrados que separan a las especies estudiadas deberán utilizarse en conjunto con otros caracteres como los reproductivos (flores, frutos, semillas) para apoyar la propuesta de Trelease (1924) de reconocer a este par de especies como taxa diferentes.

\section{Agradecimientos}

Se agradece a los curadores de los herbarios CHAPA, ENCB, MEXU, XAL por las facilidades para tener acceso al material consultado y por la oportunidad de remover material para el estudio de la arquitectura foliar, al CONACYT (No. 159282) la beca otorgada a D.M.C. para realizar estudios de postgrado, a Héctor Hernández por el trabajo fotográfico de cuarto obscuro y a Susana Valencia y Guillermo Ángeles por su revisión crítica.

\section{Literatura citada}

Baas P. 1982. Systematic, phylogenetic, and ecological wood anatomy. History and perspectives. En: Baas P. Ed. New Perspectives in Wood Anatomy, pp. 23-58, Martinus Nijohoff Publishers, La Haya.

Bamber R.K. 1962. The anatomy of the barks of Leptospermoideae. Australian Journal of Botany 10:25-54.

Chalk L. 1983. The effects of ecological conditions on wood anatomy. En: Metcalfe C.R. y Chalk L. Eds. Anatomy of the Dicotyledons, pp. 152-156, Vol. II, 2a ed., Clarendon Press, Oxford.

Corral L.G. 1981. Anatomía de la madera de siete especies del género Quercus. Boletín Técnico del Instituto Nacional de Investigaciones Forestales (México) 72:1-55.

Cuttler D.F., Rudall P.J., Gasson P.E. y Gale R.M. 1987. Root Identification Manual of Trees and Shrubs. Chapman and Hall, Londres.

de la Paz P.O.C. 1974. Anatomía de la madera de cinco especies de encinos de Durango. Boletín Técnico del Instituto Nacional de Investigaciones Forestales (México) 43:1-35.

de la Paz P.O.C. 1976. Características anatómicas de cinco encinos de México. Boletín Técnico del Instituto Nacional de Investigaciones Forestales (México) 46:1-43.

de la Paz P.O.C. 1982. Estructura anatómica de cinco especies del género Quercus. Boletín Técnico del Instituto Nacional de Investigaciones Forestales (México) 88:1-62. de la Paz P.O.C. 1985. Caracteres anatómicos de siete especies del género Quercus. Boletín Técnico del Instituto Nacional de Investigaciones Forestales (México) 123:1-70.

de la Paz P.O.C. y Aguilar L. 1978. Diferencias morfológicas externas y anatómicas de la madera de encinos blancos y rojos. Boletín Técnico del Instituto Nacional de Investigaciones Forestales (México) 59:1-19.

de la Paz P.O.C, Campos A., Quintanar A. y Dávalos-Sotelo R. 1998. Estudio anatómico de la madera de cinco especies del género Quercus (Fagaceae) del estado de Veracruz. Madera y Bosques 4:45-65.

Dickison W.C. 1975. The bases of angiosperm phylogeny: vegetative anatomy. Annals of the Missouri Botanical Garden 62:590620.

Dilcher D. 1974. Approaches to the identification of angiosperm leaf remains. The Botanical Review (Lancaster) 40:1-157.

Dos Santos G. y Miller R.B. 1998. Wood anatomy of Jacaranda (Bignoniaceae): relationships in sections Monolobos and Dilolobos as suggested by twig and stem wood rays. International Association of Wood Anatomists Journal 18:369-384.

Hardin W.J. 1979. Patterns of variation in foliar trichomes of eastern North American Quercus. American Journal of Botany 66:576-585.

Hickey L. 1973. Classification of the architecture of dicotyledonous leaves. American Journal of Botany 60:17-33.

Hickey L. 1974. Clasificación de la arquitectura foliar en dicotiledóneas. Boletín de la Sociedad Argentina de Botánica 16:1-26.

Hickey L. y Wolfe J.A. 1975. The bases of angiosperm phylogeny: vegetative morphology. Annals of the Missouri Botanical Garden 62:538-589.

Howard E.T. 1977. Bark structure of southern upland oaks. Wood and Fiber 9:172-183.

IAWA (International Association of Wood Anatomists) Committee. 1989. List of microscopic features for hardwood identification. International Association of Wood Anatomists Bulletin New Series 10:219-332.

Johansen J. 1940. Plant Microtechnique. McGraw-Hill Book Co., Nueva York.

Junikka L. 1994. Survey of English microscopic bark terminology. International Association of Wood Anatomists Journal 15:3-45.

Llamas F., Pérez-Morales C., Acedo C. y Penas A. 1995. Foliar trichomes of the evergreen and semi-deciduous species of the genus Quercus (Fagaceae) in the Iberian Peninsula. Botanical Journal of the Linnean Society 117:47-57.

Manos S.P. 1993. Foliar trichome variation in Quercus section Protobalanus (Fagaceae). Sida 15:391-403.

Martínez-Cabrera D., Terrazas T. y Zavala-Chávez F. En prensa. Estudio morfométrico de Quercus sartorii y Q. xalapensis (Fagaceae). Madroño.

Nixon K.C. 1989. Phylogeny and systematics of the oaks. New York Food and Life Sciences Quarterly 22:7-10.

Nixon K.C. 1993. Infrageneric classification of Quercus (Fagaceae) and typification of sectional names. Annales des Sciences Forestières 50, Suppl. 1:25S-34S.

Orduño-Cruz A. y Terrazas T. 1998. Anatomía de la corteza de tres especies de leguminosas I. Origen y desarrollo. Boletín de la Sociedad Botánica de México 63:51-65.

Panshin A.J. y de Zeeuw C. 1980. Textbook of Wood Technology. 4a ed. McGraw-Hill-Book, Nueva York. 
Ritcher H.G. y Van Wyk A.E. 1990. Wood and bark anatomy of Lauraceae IV: Dahlgrenodendron J.H. Van der Merwe y Van Wyk. International Association of Wood Anatomists Bulletin, New Series 11:173-182.

Romero R.S. 2000. Estudio taxonómico de la serie Acutifoliae (Quercus, Fagaceae). Tesis doctoral, Facultad de Ciencias, Universidad Nacional Autónoma de México, México, D.F. 174 pp.

Romero R.S., Lira R. y Dávila P. 2000. A phenetic study of the taxonomic delimitation of Quercus acutifolia and Q. conspersa (Fagaceae). Brittonia 52:177-187.

Roth I. 1981. Structural Patterns of Tropical Barks. Encyclopedia of Plant Anatomy IX, part 3. Gebruder Borntraeger, Berlín.

Rzedowski J. 1978. Vegetación de México. Limusa, México, D.F.

SAS Institute. 1989. User's Guide, release 6.04. SAS Institute Inc., Cary.

Stuessy T.F. 1990. Plant Taxonomy: The Systematic Evaluation of Comparative Data. Columbia University Press, Nueva York.

Terrazas T. 1995. Anatomía sistemática de la familia Anacardiaceae en México. I. La corteza de Tapirira Aublet. Boletín de la Sociedad Botánica de México 57:103-112.

Terrazas T. y Wendt T. 1995. Systematic wood anatomy of the genus Tapirira Aublet (Anacardiaceae) -a numerical approach. Brittonia 47:109-129.

Trelease W. 1924. The American Oaks. Memoirs of the National Academy of Science. Washington, D.C.

Trockenbrodt M. 1990. Survey and discussion on the terminology used in bark anatomy. International Association of Wood Anatomists Bulletin, New Series 11:141-166.

Trockenbrodt M. 1995a. Calcium oxalate crystals in the bark of the
Quercus robur, Ulmus glabra, Populus tremula and Betula pendula. Annals of Botany 75:281-284.

Trockenbrodt M. 1995b. Structure and identification of root bark of Quercus robur L. Trees 9:341-347.

Trockenbrodt M. y Parameswaran M. 1986. A contribution to the taxonomy of the genus Inga Scop. (Mimosaceae) based on the anatomy of the secondary phloem. International Association of Wood Anatomists Bulletin, New Series 7:62-71.

Valencia A.S. y Delgado A. 1995. Arquitectura de hojas como una herramienta taxonómica en el reconocimiento de individuos de encinos pertenecientes a Quercus affinis Scheidw. y Q. laurina Humb. \& Bonpl. En: Marroquín de la F. J.S. Ed. III Seminario Nacional sobre Utilización de Encinos, pp. 850-859, Universidad Autónoma de Nuevo León, Linares.

Valencia A.S. y Barajas-Morales J. 1995. Comparación de la anatomía de la madera de Quercus affinis y $Q$. laurina (Fagaceae). Anales del Instituto de Biología, Universidad Nacional Autónoma de México, Serie Botánica 66:113-131.

Van Wyk A.E. 1985. The genus Eugenia (Myrtaceae) in Southern Africa: structure and taxonomic value of bark. South African Journal of Botany 51:157-180.

Whitmore C.T. 1962. Studies in systematic bark morphology III. Bark taxonomy in Dipterocarpaceae. Gardens Bulletin Singapore 19:321-371.

Whitmore C.T. 1963. Studies in systematic bark morphology IV. The bark of beach oak and sweet chestnut. The New Phytologist 62:161-169.

Zavala-Chávez F. 1995. Encinos Hidalguenses. Ediciones Universidad Autónoma de Chapingo, Chapingo.

Fecha de recepción: 20 de agosto de 2003

Versión corregida: 28 de noviembre de 2003

Aceptado: 1 de diciembre de 2003 
Apéndice 1. Material adicional empleado para el estudio de la arquitectura foliar.

Quercus sartorii; HIDALGO: Mpio. Jacala, J. Rzedowski 23167, 23168 (ENCB); Mpio. Molango, L. González Q. 1600 (ENCB); Mpio. Zimapán, H. Puig 4413 (ENCB), R. Hernández M. 6563 (XAL). PUEBLA: Mpio. Nanzontla, F. Simón M. y A. Colín 154 (ENCB). QUERÉTARO: Mpio. Landa, H. Puig 3618 (ENCB). SAN LUIS POTOSÍ: Mpio. San Nicolás de Tolentino, J. Rzedowski 11316 (ENCB); Mpio. Tamasopo, H. Puig 3645 (ENCB); Mpio. Xilitla, J. Rzedowski 12386 (ENCB). TAMAUliPAS: Mpio. Gómez Farías, A. Gómez Pompa 2014 (MEXU), J. Rodney 292, 522 (MEXU), P. S. Martín y C. Saravia 1205 (MEXU); Mpio. Jaumave, M. Castañeda 2760 (MEXU). VERACRUZ: Mpio. Banderilla, M. G. Zola B. 531 (XAL); Mpio. Coatepec, J. I. Calzada 2008 (XAL); Mpio. Cosautlán, R. Arriaga C. 199 (XAL); Mpio. Huatusco, J. Rzedowski 18931 (MEXU); Mpio. Ixhuacán de los Reyes, R. Arriaga C. y H. López 232 (MEXU), M. Nee 22475 (ENCB); Mpio. Las Minas, C. Durán E. 1082 (MEXU); Mpio. Naolinco, F. Ventura A. 8919 (ENCB); Mpio. de Tlalnehuayocan, M. G. Zola B. 809 (XAL); Mpio. de Totutla, C. Durán y T. Platas 1182 (XAL); Mpio. Xalapa, M. G. Zola B. 6500 (MEXU); Mpio. Xilotepec, J. Dorantes y M. Acosta 1762 (ENCB); M. G. Zola B. 435 (XAL).

Quercus xalapensis; CHIAPAS: Mpio. Motozintla de Mendoza, D. E. Breedlove 42675 (ENCB). HIDALGO: Mpio. Lolotla, L. Vela G. 226 (ENCB); Mpio. Tenango de Doria, R. Hernández M. 3325 (ENCB); Mpio. Zacualtipán, J. Rzedowski y R. Madrigal 29460 (ENCB). SAN LUIS POTOSÍ: Mpio. Cd. del Maíz, J. Rzedowski 8336 (ENCB); Mpio. Tamasopo, J. Rzedowski 10628 (ENCB), J. Flamand 8 (ENCB), K. Roe 2307 (ENCB). VERACRUZ: Mpio. Chiconquiaco. M. Cházaro 3574 (MEXU); Mpio. Huayacocotla, J. Palma 159 (XAL); Mpio. Huatusco, J. Rzedowski 18931, 18938 (ENCB); Mpio. Xalapa, A. P. Vovides 60, (ENCB), M. Nee 33068 (XAL), F. Ventura A. 2361 (ENCB); Mpio. Xilotepec, J. Dorantes, M. Acosta y A. Calles 01762 (CHAPA). 